\title{
LA SEGUNDA CONFERENCIA MUNDIAL DE EDUCACIÓN SUPERIOR (UNESCO, 2009) Y LA VISIÓN DEL CONCEPTO DE ACREDITACIÓN EN LAS CONFERENCIAS DE UNESCO (1998-2009)
}

\author{
Francisco López SEgrera*
}

\begin{abstract}
*Doctor en Estudios Latinoamericanos e Hispánicos (Paris VIII, Sorbonne). Asesor Académico de la Global University Network for Innovation (GUNI) en la UPC, Barcelona. Tarragona, España. E-mail: francisco. lopez-segrera@upc.edu
\end{abstract}

Resumen: El presente artículo formula algunas reflexiones sobre la Segunda Conferencia Mundial de Educación Superior (UNESCO, 2009). El debate principal en ella versó sobre la visión de enseñanza superior como "un bien común", o como "un servicio público", esta última visión fue considerada por muchos delegados como un camino hacia una mayor mercantilización. En el Comunicado Final prevaleció el primer criterio. También en el artículo se analizan los cambios principales en los conceptos de evaluación, calidad, garantía de la calidad y acreditación entre 1995 y 2009. El énfasis se ha movido de la evaluación y la calidad (1995-1998) a la garantía de calidad y acreditación (1998-2003) y luego (2008-2009) en vincular los criterios de calidad a la pertinencia.

Palabras clave: Conferencia Mundial de Enseñanza superior de UNESCO. Evaluación, Acreditación, Garantía de calidad, Equidad.

THE SECOND WORLD CONFERENCE OF HIGHER EDUCATION (UNESCO, 2009)

AND THE VISION OF ACCREDITATION IN THE CONFERENCES OF UNESCO (1998-2009)

Abstract: The present article formulates some reflections on The Second World Higher Education Conference (UNESCO, 2009). The main debate was about the vision of higher education as "a common good", or as "a public service", this last vision was considered by many delegates as a road to further mercantilization. In the Final Communiqué the first criterion prevailed. The article also analyzes the main changes concerning the concepts of evaluation, quality, quality assurance and accreditation between 1995 and 2009. The emphasis has moved from evaluation and quality (1995-1998) to quality assurance and accreditation (1998-2003) and then (2008-2009) in linking the quality criteria to pertinence, relevance, equity, and sustainable development.

Key words: UNESCO Second World Conference of Higher Education (2009). Evaluation, Accreditation, Quality Assurance, Equity.

\section{LA CONFERENCIA MUNDIAL SOBRE EDUCACIÓN SUPERIOR 2009 (CMES)}

La Conferencia Mundial sobre Educación Superior 2009 (CMES) se celebró en la sede de la UNESCO en París del 5 al 8 de julio de 2009. En ella se analizaron los cambios desarrollados en la educación superior desde la primera Conferencia Mundial de 1998 y se centró en "Las Nuevas Dinámicas de la Educación Superior y de la Investigación para el Cambio Social". 
La crisis civilizatoria actual implica la construcción de nuevos paradigmas con el fin de transitar hacia una sociedad planetaria sostenible. Este proceso afecta también a la educación superior. Una de las tareas prioritarias para las instituciones de educación superior (IES) es poner a disposición el conocimiento existente y generar conocimiento nuevo al servicio de la construcción social. Esto sin duda tiene implicaciones enormes para la educación superior en su conjunto y para sus instituciones.

En términos globales, la matriculación mundial ha pasado de 92 millones en 1999, con 44,2 millones de mujeres, a 150,7 millones en el 2007, con 76,8 millones de mujeres. Esta expansión masiva de la educación superior se ha visto además marcada por factores como: la incapacidad de los estados para financiar este crecimiento; el aumento de la educación superior privada y la emergencia de nuevos proveedores mayoritariamente 'con ánimo de lucro'; la diversificación de las fuentes de ingreso y los mecanismos de distribución de costes; la internacionalización y la provisión transfronteriza; y la importancia de la acreditación para la garantía de la calidad y de los rankings. Todos estos temas se debatieron en la CMES 2009.

La idea de bien público en la educación superior está directamente relacionada con los roles que las IES desempeñan en la sociedad. Es en ellas donde se forma a las personas que alcanzarán las posiciones de mayor responsabilidad en la sociedad. Por tanto, la educación superior tiene una responsabilidad pública fundamental respecto a los contenidos curriculares, la ética y los valores que transmite. La reflexión sobre la contribución y responsabilidad social de las universidades implica una revisión a fondo de su misión.

Por otra parte, es necesario equilibrar el conocimiento económicamente pertinente con el conocimiento humano y social pertinente. La educación superior puede ser eficaz y eficiente en términos económicos sin ser socialmente pertinente. Es necesaria la pertinencia académica y la social.

La Conferencia ofreció una plataforma global para el debate y el análisis con visión de futuro de la educación superior y la investigación, y alcanzó acuerdos sobre recomendaciones orientadas a la acción que permitirán que la enseñanza superior y la investigación respondan mejor a las nuevas dinámicas que formarán la agenda para el desarrollo de las políticas e instituciones de educación superior. La Conferencia ratificó la importancia de la educación superior y la investigación para hacer frente a los desafíos mundiales y construir economías basadas en el conocimiento que sean más integradoras, equitativas y sostenibles.

Previó a la CMES 2009 se organizaron reuniones regionales preparatorias con el fin de formular propuestas regionales concretas para la Conferencia Mundial de 2009. Estas reuniones se celebraron en: 
a) América Latina y el Caribe, CRES 2008 (Colombia, Cartagena, 4 al 6 de junio de 2008).

b) La Reunión Preparatoria Subregional de Asia y el Pacífico se celebró del 25 al 26 de septiembre de 2008 en Macao (China). ${ }^{1}$

c) Se celebró en Nueva Delhi (India), el 25 y 26 de Febrero de 2009 una segunda conferencia sub-regional en Asia.

d) La Conferencia Regional de Educación Superior de África se celebró en Dakar (Senegal), del 10 al 13 de noviembre de 2008.El Fórum UNESCO de Educación Superior en la Región Europea tuvo lugar en Bucarest, Rumanía, del 21 al 24 mayo del 2009.

e) La Conferencia Regional Árabe de Educación Superior se celebró en el Cairo, Egipto, del 31 mayo al 2 de junio de 2009.

Todas estas Conferencias Regionales emitieron Declaraciones Finales o documentos similares con los acuerdos alcanzados y esto sirvió como base para el debate en la CMES 2009

La CMES 2009 contó con unos 1200 participantes distribuidos del modo siguiente:

- Delegados de los Estados Miembros de la UNESCO;

- Representantes de los organismos de las Naciones Unidas y otras organizaciones intergubernamentales;

- Representantes de ONG y de la sociedad civil;

- Participantes del sector privado, y

- Expertos que asistieron a título individual.

La conferencia se centró en tres temas principales:

- La función de la educación superior en el tratamiento de los grandes desafíos mundiales.

- Compromiso de la sociedad y responsabilidad social con la educación superior.

- Fomentar la excelencia para acelerar el desarrollo de África: "Hacia una esfera africana de educación superior e investigación".

1 En Asia dada la extensión y diversidad de la región se celebró una segunda conferencia preparatoria en India. 
Otros temas que debatidos en las sesiones plenarias fueron: De 1998 a 2009, y más allá: Las Nuevas Dinámicas de la Educación Superior y de la Investigación; Más allá de las palabras: ¿Qué acciones emprender en la Educación Superior y en la Investigación? ¿Qué rumbo pueden seguir la Educación Superior y la Investigación?

En las sesiones paralelas se debatieron 3 subtemas:

- Internacionalización, regionalización y mundialización

- $\quad$ Equidad, acceso y calidad

- Aprendizaje, investigación e innovación

Y también las Nuevas Dinámicas de la educación superior:

- Demanda

- Diversificación

- Creación de redes

- Aprendizaje a lo largo de toda la vida

- Tecnologías de la información y la comunicación

- Responsabilidad social

- Evolución del papel de los gobiernos

Además, durante la conferencia tuvieron lugar cinco eventos especiales. Una sesión especial de GUNI fue uno de estos eventos.

En la inauguración de la CMES 2009, el Director General de UNESCO, recordó a los más de mil delegados de 70 países reunidos en París, que el tema de esta Conferencia son las nuevas dinámicas de la educación superior y la investigación para el cambio social y el desarrollo.

Eso refleja dos creencias. La primera es que las nuevas fuerzas están transformando la educación superior a una velocidad que no se habría podido prever hace diez años. Y la segunda es que se reafirma la convicción de que la forma en que conduzcamos la educación superior va a determinar cómo vamos a responder a esas nuevas fuerzas.

Dijo Matsuura. Destacó como nuevas dinámicas relevantes la expansión de las tasas de matrícula, las TIC y la globalización.

Los discursos inaugurales introdujeron los temas principales de la conferencia. El discurso dominante en la Conferencia fue el papel que podría 
desempeñar la enseñanza superior para que concluyese la recesión global y qué medidas tomar para que esta crisis no la afectase. Esto fue acompañado por declaraciones sobre la ES como bien público (a diferencia de un servicio público); la necesidad de la equidad en el acceso y de la excelencia; significado de la crisis de valores; la necesidad de inversión sostenida en ES (de los estados nacionales, de las organizaciones internacionales como el Banco Mundial y UNESCO, de organizaciones multilaterales y de otros donantes); sociedades públicas/privadas para el desarrollo de la ES ; libertad de cátedra y autonomía institucional; la necesidad de sistemas de garantía de calidad globalizados, como protección ante los nuevos proveedores ; la necesidad de desarrollar las TIC; de innovación en métodos y contenidos; importancia del desarrollo de redes de investigación y de construcción de capacidades entre países desarrollados y en desarrollo; y posibilidades de empleo para los graduados.

Los informes recibidos de las 6 conferencias regionales preparatorias (Macao, Cartagena, Bucarest, El Cairo, Dakar, y Nueva Deli) enfatizaron temas comunes:

- La ES derecho humano y un bien público social

- Tasas de participación, Acceso y Equidad

- Valores sociales y humanos de la ES

- Fuga de Cerebros y pérdida de capital humano en los países de menores ingresos

- Redes Académicas

- Integración Regional y la creación de áreas de ES como Bologna

- Participación de los grupos de interés y de las comunidades en la ES

- Desarrollo de las TIC

- Participación del sector Privado en la ES

- Relevancia

- Eficiencia y efectividad

- Garantía de calidad

- Investigación e Innovación

- Competitividad 
- Planificación Estratégica

- Buen Gobierno

- Movilidad académica

- Lucha contra la pobreza

En la mesa redonda sobre África se destacó que 53 países de esa región no alcanzarían los Objetivos del Milenio de ONU, y que solo una universidad en la RSA está entre las mejores 100.

Muchos expertos consideraron que las intervenciones eran muy desiguales, junto a algunas excelentes hubo muchas que repitieron lugares comunes. Algunos expertos de especial relevancia no tuvieron participación en la Conferencia como ponentes y los miembros del Foro UNESCO de educación superior no fueron invitados en su condición de tales.

El discurso de los participantes de los países desarrollados tuvo en algunos casos un acento neo-liberal, a diferencia del discurso crítico que predomino en las intervenciones de los participantes de América Latina y el Caribe y de África.

Uno de los debates de mayor relevancia con el fin de alcanzar un consenso en el Comunicado Final fue el planteamiento del Grupo Latinoamericano (GRULAC) a través de sus Ministros de Educación y Jefes de Delegaciones con relación a incluir en dicho Comunicado los acuerdos principales de la CRES 2008. En especial exigieron ante la II Conferencia Mundial que quedase plasmado en el Comunicado Final que la educación superior es un bien público social, y que es un deber de los Estados garantizarlo como prioridad en la nación. Tras un largo debate a lo largo de la CMES 2009 se incluyó el concepto de la educación superior como bien público en el Comunicado Final y no como servicio público, criterio que defendían otros participantes.

Hubo aspectos positivos con relación a la CMES de 1998 como fue una mayor presencia de redes y una visión más global de las diversas regiones y menos centradas en los problemas de un solo país o universidad, una mayor unidad latinoamericana, un mayor consenso entre latinoamericanos y africanos, un consenso en torno a temas claves como la sostenibilidad y el evitar reducir el financiamiento y el acceso a la educación superior pese a las presiones a que somete a los países, y en especial a los de menor desarrollo, la crisis económica actual. El Programa en sus distintas sesiones cubrió los temas claves en el debate de la educación superior y lo expresado en las diversas declaraciones y planes de acción regionales. También los documentos 
y presentaciones tuvieron una elevada calidad e igualmente el debate a lo largo de la Conferencia. Dedicar una sesión plenaria a una mesa redonda sobre África y otra al tema de responsabilidad social fue considerado muy positivo por los participantes.

El director adjunto de IESALC, José Renato Carvalho, destacó que este tipo de encuentros son siempre una oportunidad para el diálogo y celebró que muchos de los principios que promueve la región en torno a la educación superior fueron reflejados en la declaración final de la Conferencia. Un importante logro de la Conferencia fue mantener el concepto de la educación superior como un bien público y un derecho humano fundamental, como se acordó en la Conferencia Regional de Educación Superior, en octubre de 2008, en Cartagena.

En lo que se refiere a los aspectos negativos Axel Didriksson afirmó en una entrevista que le hizo IESALC durante la Conferencia lo siguiente: "Se siguen reiterando las mismas cosas, los mismos conceptos, no se avanza y, sobre todo, no se toman medidas. De diez años para acá, las reflexiones no se ven acompañadas, como lo muestran algunos participantes, con acciones concretas de la UNESCO. Por eso, uno de los temas que pusimos y que queremos que se subraye desde la región de América Latina y el Caribe, es que se adopten normas, acciones de gobierno y recomendaciones muy fuertes alrededor de la Responsabilidad Social, la Pertinencia y de una nueva cooperación internacional que busque equilibrio entre las regiones para evitar las grandes brechas que todavía padecemos".

Varios participantes latinoamericanos como Rafael Guarga, Secretario General del Grupo Montevideo y Axel Didriksson se refirieron a la muy escasa representación de América Latina y de las propuestas de la CRES 2008. Según Didriksson:

se puede observar que, por ejemplo, hubo mayor promoción en la participación del continente africano. No digo que no la haya, al contrario, hay que apoyarlos porque están en una situación muy complicada. Pero la parte latinoamericana estaba muy restringida. Incluso hay observaciones de muchos colegas con respecto a las limitaciones de la participación de algunas delegaciones latinoamericanas en esta Conferencia. Está más representada Europa, está más representada África, están menos representadas Asia y América Latina. Son desbalances regionales que no debemos permitir.

En la sesión de Clausura de la Conferencia se dio a conocer el Borrador del Comunicado Final en cuyo preámbulo se afirma: 
La Educación Superior, como un bien público y un imperativo estratégico para todos los niveles de educación y como la base para la investigación, la innovación, y la creatividad, debe ser un asunto de responsabilidad y tener el apoyo económico de todos los gobiernos. Como afirma la Declaración Universal de Derechos humanos, "la enseñanza superior será igualmente accesible a todos sobre la base del mérito" (Art. 26, el párrafo 1).

Las otras partes del Comunicado analizan: la responsabilidad social de la educación superior; acceso, equidad y calidad; internacionalización, regionalización y globalización; aprendizaje, investigación e innovación; la educación superior en África; un llamado a la acción por parte de los Estados Miembros; y un llamado a la acción por parte de UNESCO.

La II Conferencia Mundial rescató además el mensaje de la I Conferencia Mundial, que se celebró en 1998, al indicar que "la década que acaba de concluir evidenció que la educación superior y la investigación contribuyen a erradicar la pobreza, al desarrollo sostenible y al progreso hacia los objetivos de desarrollo acordados internacionalmente".

Los delegados reunidos en París hicieron un llamado a sus gobiernos para que se mantenga y, en la medida de lo posible, se incremente, la inversión en educación superior "con el objetivo de mantener la calidad y la igualdad en todo momento y promover la diversificación, tanto en garantizar el acceso de educación superior como en su financiamiento".

En las acciones que esperan de la UNESCO, los participantes de la Conferencia, la exhortan a promover la movilidad y los intercambios de estudiantes y académicos y, al mismo tiempo, desarrollar estrategias para evitar la fuga de cerebros.

Esta Conferencia representa un verdadero hito, en el que se han encontrado más de mil delegados de unos 150 países. Se exploró una variedad muy amplia de temas y se debatieron asuntos bastante polémicos. Algunas veces estuvimos de acuerdo y otras no. Pero por encima de todo estos, todos estamos unidos en torno a la importancia de la Educación Superior, (dijo el Director General de la UNESCO, Koïchiro Matsuura, en la sesión de clausura).

Los delegados acordaron también manifestar su apoyo a la decisión de que el foco de esta Conferencia Mundial estuviera centrado en África, el continente donde crece más rápido la matriculación en educación superior, pero también en el que menos porcentaje de la población tiene acceso a ella. 
La Conferencia Mundial de la UNESCO sobre Educación Superior no tiene, como es conocido, una definición normativa para que sus conclusiones puedan ser acatadas por los Estados miembros, porque se trata sólo de recomendaciones. Aunque no siempre estas recomendaciones y acuerdos se plasman en legislaciones y planes de acción en los diversos Estados miembros de UNESCO, sin duda la CMES 2009 marca un hito altamente positivo con relación a una educación superior que contribuya en forma decisiva a la erradicación de la pobreza y al desarrollo sostenible. La Nueva Agenda Educativa de los países tendrá en cuenta los logros de esta II Conferencia Mundial de UNESCO sobre la educación superior.

\section{VISIÓN DE LA ACREDITACIÓN EN LAS CONFERENCIAS DE UNESCO (1998-2009)}

El principal cambio en el enfoque de los conceptos interrelacionados de evaluación, calidad, garantía de la calidad y acreditación es que el énfasis ha pasado de estar en la evaluación y la calidad (1995-1998) durante las Conferencias Regionales de UNESCO preparatorias de la Conferencia Mundial de Educación Superior (CMES, 1998) a estar en la garantía de la calidad y la acreditación (1998-2003) en el proceso de "seguimiento" de la CMES. En la CRES 2008 y en la CMES 2009 lo nuevo consistió en vincular los criterios de calidad a la pertinencia, la equidad y el desarrollo sostenible y no solo a su eficiencia, visualizando la educación superior como "un bien público social, un derecho humano y universal y un deber del Estado" (CRES, 2008).

El Documento de Política para el Cambio y el Desarrollo en la Educación Superior (1995) de la UNESCO inició el proceso que llevó a las conferencias regionales (La Habana, 1996; Dakar, Tokio, Palermo, 1997, Beirut, 1998) y a la Conferencia Mundial sobre la Educación Superior (WCHE, por sus siglas en inglés, 1998). En este documento de política, la calidad se considera una «preocupación fundamental» en el ámbito de la educación superior. La evaluación también se considera fundamental para fomentar la calidad del personal, los programas y el proceso de aprendizaje. Sin embargo, este documento no se refiere a la acreditación y la garantía de la calidad como un resultado evidente del proceso de evaluación de la calidad (UNESCO, 1995).

Ahora analizaremos los diferentes enfoques de calidad de las conferencias regionales mencionadas. En la Declaración sobre la Educación Superior en América Latina y el Caribe (La Habana), "la necesidad de participar firmemente en la mejora cualitativa de todos los niveles del sistema educativo" se consideró "uno de los desafíos que ha planteado este nuevo siglo". 
Sus contribuciones más concretas pueden hacerse realidad a través de: la formación de profesores; la transformación de los estudiantes en agentes activos de su formación; la promoción de la investigación socioeducativa de problemas como el abandono escolar prematuro y la repetición de curso, y la garantía de su contribución al diseño de las políticas estatales en materia de educación. (UNESCO, 1998a)

La Declaración y plan de acción sobre la educación superior en África (Dakar), recomendaba que los estados miembros: "establezcan un mecanismo para evaluar la calidad de las instituciones de educación superior" y expresaba la "esperanza de que todas las instituciones creen estructuras adecuadas para evaluar y controlar la calidad de sus planes de estudios (incluido el rendimiento de los estudiantes)".

En la Declaración sobre la educación superior en Asia y el Pacífico (Tokio), la calidad se considera

un concepto multidimensional y no es posible llegar a un conjunto de criterios estándar de la calidad aplicables a todos los países y con los que se pueda evaluar a las instituciones. La calidad abarca las principales funciones y actividades de la educación superior: enseñanza y programas académicos, investigación y becas, contratación de personal, estudiantes, infraestructura y entorno académico. Tanto la evaluación interna como la revisión externa son componentes cruciales de cualquier sistema de garantía de la calidad bien desarrollado. El concepto de rendición de cuentas va estrechamente unido al de calidad.

El Plan de acción recomienda "la renovación del plan de estudios" para mejorar la calidad. Según este Plan: "Los países deben introducir métodos de garantía de la calidad tanto en el ámbito institucional como en el sistémico. Entre ellos, pueden figurar la acreditación académica, las auditorías académicas y las evaluaciones institucionales".

El Foro Regional Europeo (Palermo) reconoció que:

Dado el aumento de la demanda de educación superior y su democratización, existe una apremiante necesidad de compartir buenas prácticas y de garantizar los criterios estándar de la calidad académica mediante una cultura de la calidad y los instrumentos para la garantía de la calidad tanto en el ámbito sistémico como en el institucional.

La Declaración de Beirut sobre la educación superior en los estados árabes declaró que: 
Todos los sistemas de educación superior y las instituciones deberían dar prioridad a garantizar la calidad de los programas, la enseñanza y los resultados. Las estructuras, los procedimientos y los criterios estándar para garantizar la calidad deberían desarrollarse en el ámbito regional y nacional con arreglo a las pautas de actuación internacionales, a la vez que respeten la variedad en función de las características específicas de cada país, institución o programa. Además, las instituciones de educación superior necesitan los recursos económicos y humanos adecuados para aumentar la calidad de la educación.

Las declaraciones de Dakar, Tokio y Beirut presentan conceptos de la calidad que también están en consonancia con las definiciones establecidas en el Documento de política para el cambio y el desarrollo en la educación superior de la UNESCO. Una suposición básica es que la calidad está vinculada a la relevancia (la calidad no tiene sentido sin la relevancia, tal como se estipula en la declaración de los estados árabes). Además, la calidad es un concepto multidimensional que depende en gran medida del contexto en el que se encuentra un determinado sistema, una misión institucional o las condiciones y los criterios estándar en el seno de una disciplina específica. Teniendo esto presente, la Declaración de Tokio afirma que, evidentemente, "no es posible llegar a un único conjunto de criterios estándar de calidad aplicable a todos los países". El concepto de rendición de cuentas va estrechamente unido al de la calidad (Tokio), y es necesaria una evaluación continua para cumplir este objetivo (Tokio).

La Declaración de Dakar incluye la idea de que la calidad "comporta la operacionalización de los resultados previstos (una clara definición de los fines y objetivos), de los insumos con que trabajarán las instituciones (una revisión de los criterios de admisión) y de los procesos y procedimientos para trabajar con los insumos (el modo en que el sistema de gestión coordina las estructuras, los recursos y la cultura institucional para obtener el producto requerido)". En el documento de Palermo, la investigación parece fundamental para la calidad y la efectividad de toda la educación superior.

En la Declaración de La Habana, "las redes para relacionar instituciones, profesores y estudiantes son una cuestión clave en la búsqueda colectiva de la equidad, la calidad y la relevancia en la educación superior". (UNESCO, 1998b).

En la Declaración mundial sobre la educación superior en el siglo XXI: visión y acción (octubre de 1998), en el artículo 11 sobre evaluación cualitativa, se considera que: 
La calidad de la educación superior es un concepto multidimensional que debería comprender todas sus funciones y actividades: la enseñanza y los programas académicos, la investigación y los estudios, la contratación de personal, los alumnos, los edificios, las instalaciones, los equipamientos, los servicios a la comunidad y el entorno académico. La autoevaluación interna y la revisión externa, realizadas abiertamente por especialistas externos, si es posible con experiencia internacional, son vitales para mejorar la calidad.

La dimensión internacional de la calidad, la selección del personal y su formación continua, y la obtención de experiencia en TIC también se consideran aspectos clave para la evaluación de la calidad.

El Marco de acción prioritaria para el cambio y el desarrollo de la educación superior (octubre 1998) recomendaba a las instituciones de educación superior que una prioridad fuera "garantizar la alta calidad de la posición internacional, considerar la rendición de cuentas y la evaluación tanto interna como externa, con el debido respeto de la autonomía y la libertad académica, inherentes a su operativa".

Sin embargo, los conceptos de garantía de la calidad y de acreditación no se mencionan en ninguno de estos documentos ni tampoco en las declaraciones regionales o en la declaración mundial como una continuación ni como resultado de la evaluación. El énfasis se pone en la calidad, no en la acreditación como consecuencia natural. De hecho, el concepto de garantía de la calidad sólo se menciona en las declaraciones regionales europeas, árabes y asiáticas. El concepto de acreditación sólo se menciona en la declaración de Tokio.

En junio de 2003, cinco años después de la WCHE, la UNESCO reunió a los actores clave en el ámbito de la educación superior de todas las áreas de París. Estos actores representaban los puntos centrales de la WCHE y eran responsables del seguimiento de la conferencia.

Ahora analizaremos sus puntos de vista respecto a la calidad y la acreditación, y el esfuerzo realizado durante el período 1998-2003 para aplicar las recomendaciones de la WCHE.

En el documento relativo a América Latina y el Caribe, preparado por el Instituto Internacional para la Educación Superior en América Latina y el Caribe (IESALC), "el desarrollo de sistemas nacionales de evaluación y acreditación de la educación superior" se consideraba uno de los principales aspectos en los que los cambios habían sido considerables.

Se implantaron sistemas nacionales de evaluación y acreditación de ámbito nacional principalmente en Argentina, Cuba, Brasil, Colombia, Chile, Costa 
Rica y México y esto se extendió gradualmente al resto de los países de la región (IESALC, 2003).

En África, el deterioro de las infraestructuras, la fuga de cerebros y la gran cantidad de vacantes en puestos universitarios son algunos de los principales factores que dificultan la mejora de la calidad. En Ghana, en 1998, el $40 \%$ de las plazas universitarias estaban vacantes, al igual que el $60 \%$ de las plazas de escuelas técnicas (UNESCO, 2003a).

El Informe regional de Asia y el Pacífico mencionaba que «muchos estados miembros han establecido o reforzado recientemente sus mecanismos nacionales para garantizar la calidad en la educación superior, a la vez que se han esforzado por desarrollar nuevos indicadores de calidad». Según este informe: "La garantía de la calidad en la educación superior puede definirse como la gestión sistemática y los procedimientos de evaluación para supervisar el rendimiento de las instituciones de educación superior." Y añade:

El alcance de la innovación en los sistemas de garantía de la calidad de toda la región es impresionante. Incluso países con sistemas bien desarrollados - Australia, Corea, India, Nueva Zelanda y Malasia - han seguido realizando cambios, mientras que en otros casos los países han ensayado proyectos piloto, o se están embarcando en nuevas iniciativas importantes.

En el año 2002, un taller de trabajo regional organizado por la UNESCO de Bangkok y el Consejo Nacional de Evaluación y Acreditación (NAAC, por sus siglas en inglés) de la India se propuso desarrollar indicadores de calidad institucional y fomentar la colaboración en red para garantizar la calidad en la región. Este tipo de indicador también se ha desarrollado en Australia e Indonesia. Además, otros países han probado el benchmarking como instrumento de autoevaluación. El informe también mencionaba lo siguiente: "Los nuevos mecanismos de garantía de la calidad necesitan la inversión de más recursos, tanto sistémicos como institucionales" (UNESCO, 2003b).

El Informe Regional sobre Europa puso de relieve que el nuevo resultado obtenido a partir de la garantía de la calidad era el "surgimiento de la acreditación". Ésta se definió como la confirmación por parte de un organismo externo de que los estándares claves de la calidad estaban garantizados en instituciones y programas de educación superior determinados (UNESCO; CEPES, 2003).

El Documento sobre la educación superior en la región árabe (1998-2003) mencionaba que, hasta la Conferencia Árabe sobre la Educación Superior (Beirut, 1998), Jordania era el único estado árabe que había establecido un 
organismo que se ocupara de la acreditación. El Comité Nacional de Acreditación se fundó en 1996. Después de la Conferencia de Beirut, la Asociación de Universidades Árabes (AARU, por sus siglas en inglés) estableció un Comité Regional de Evaluación y Acreditación Universitaria. La UNESCO participa activamente en este Comité. Celebró su primera reunión en Ammán, Jordania, en el año 2001. Otros países de la región han seguido el ejemplo de Jordania estableciendo organismos similares. Estos países abarcan, entre otros: Argelia, Egipto, Líbano, Marruecos, Omán, Arabia Saudí, los Emiratos Árabes Unidos, Yemen y la Autoridad Palestina (UNESCO, 2003c).

En la CRES 2008 se consideró que la calidad debía estar "vinculada a la pertinencia y la responsabilidad con el desarrollo sostenible de la sociedad" (CRES, 2008).

En la CMES 2009 se afirmó que "la educación superior debe tratar de alcanzar simultáneamente los objetivos de equidad, pertinencia y calidad" (UNESCO, 2009).

Hubo varios factores convergentes que hicieron de la garantía de la calidad y la acreditación temas de suma actualidad durante el período posterior a la celebración de la CMES 1998, durante las reuniones de "seguimiento" (19981993) y durante las conferencias preparatorias y la CMES 2009. Estos factores son los siguientes: la expansión cuantitativa y la correspondiente diversificación de las instituciones; las estructuras, los programas y las modalidades de impartición; los procedimientos más formales y los reglamentos para la asignación de las partidas presupuestarias, y el aumento de la presión del mercado, que exige que las universidades y las instituciones de educación superior se esfuercen por posicionarse en contextos sumamente competitivos a escala nacional e internacional. Pero también en la segunda mitad de la década el concepto de acreditación apareció cada vez más vinculado al concepto de la educación superior como un bien público que debe ser preservado de la voracidad del mercado y tener entre sus prioridades la pertinencia, la responsabilidad social, la equidad y el desarrollo sostenible.

En todas partes existe una necesidad apremiante de disponer de mecanismos para garantizar la calidad y de procedimientos de acreditación. Se ha hecho un gran esfuerzo por establecer estos mecanismos y procedimientos en muchos países. Como se observa en el análisis anterior de los diferentes documentos regionales, el énfasis ha pasado de estar en la evaluación y la calidad como conceptos abstractos y aislados (1995-1998) a estar en la garantía de la calidad y la acreditación como resultado y como continuación del proceso de evaluación para garantizar la calidad (1998-2003), para finalmente (2008-2009) vincularse 
a valores tales como la pertinencia, la responsabilidad social, la equidad y el desarrollo sostenible.

La acreditación ha adquirido gran importancia, sobre todo en aquellos países y regiones (América del Sur y Caribe, los países árabes, Europa Central y Oriental, Asia y el Pacífico) donde aumenta rápidamente el número de nuevas instituciones de educación superior, sobre todo privadas.

Según la UNESCO (2003d, p. 18),

En la actualidad se usan tres tipos principales de sistemas de acreditación: $a$ ) los organismos gubernamentales; $b$ ) los organismos intermedios; $c$ ) los organismos profesionales/académicos. La tendencia actual es establecer organismos de tipo $a$ ), con un número creciente de elementos de estructuras y prácticas desarrolladas por los tipos $b$ y $c$.

Se están llevando a cabo notables esfuerzos para que la acreditación dependa cada vez menos de un organismo extranjero, aunque este sistema se sigue usando en algunos países. La experiencia demuestra que el mejor enfoque para la garantía de la calidad y la acreditación se basa en las redes regionales y en el contexto internacional. El escenario internacional facilita el establecimiento de acuerdos sobre criterios estándar y sobre el reconocimiento mutuo de títulos de grado y titulaciones, tal como se constata en el reciente proceso de Bolonia y en los esfuerzos realizados por Mercosur y en otras regiones. En consecuencia, se han creado muchas redes regionales de garantía de la calidad.

En síntesis:

Existe una necesidad evidente de tratar los temas de la garantía de la calidad, la acreditación y el reconocimiento de estudios y titulaciones en estrecha interrelación, y en un contexto internacional. El objetivo es llegar a un marco legal, que sea transparente, comúnmente aceptable y beneficioso para todos. Evidentemente, esto sólo se puede conseguir a través del diálogo y la colaboración entre las autoridades nacionales y las comunidades de educación superior. Como organismo intergubernamental en el sistema de Naciones Unidas responsable de la educación superior, la UNESCO tiene un importante papel que desempeñar, principalmente a través del Foro Mundial sobre Garantía de la Calidad y Convalidación de Títulos (UNESCO, 2003d, p. 19). 


\section{REFERÊNCIAS 2}

CMES 2009. Las nuevas dinámicas de la educación superior y de la investigación para el cambio social. Paris, 2009. Disponible en: $<\mathrm{http}: / /$ www.iesalc.unesco.org>.

GUNI 2009. Higher education at a time of transformation. New dynamics for social responsibility. Disponible en: <http://www.iesalc.unesco.org $>$.

CRES 2008. Cartagena, Colombia, 2008.

IESALC. Reformas e innovaciones en la educación superior en algunos países de América Latina y el Caribe (1998 2003). París: UNESCO, 2003.

UNESCO. Documento de política para el cambio y el desarrollo en la educación superior. París: UNESCO, 1995.

UNESCO. Declaración mundial sobre la educación superior en el siglo XXI: visión y acción. París: UNESCO, 1998a.

UNESCO. Declaraciones consolidadas y planes de acción de las conferencias regionales sobre educación superior celebradas en La Habana, Dakar, Tokio, Palermo y Beirut. Lecciones aprendidas. París: UNESCO, 1998b.

UNESCO. Special Report: state of higher education in the world today. Disponible en: $<$ http://www.iesalc.unesco.org $>$. 2009.

UNESCO. Higher education, research \& innovation: changing dynamics. Disponible en: $<$ http://www.iesalc.unesco.org $>$. 2009.

UNESCO. Institute of statistics (UIS). Trends in global higher education: tracking an academic revolution. Disponible en: $<\mathrm{http}: / / \mathrm{www}$.iesalc.unesco. org $>$.

UNESCO. Tendencias y perspectivas recientes de la educación superior en el África Subsahariana en el siglo XXI. París: UNESCO, 2003a.

UNESCO. Educación superior en Asia y el Pacífico (1998-2003). París: UNESCO, 2003b.

2 Consultar la web del IESALC http://www.iesalc.unesco.org para acceder a los documentos relacionados en la bibliografía. 
UNESCO. Educación superior en la región árabe (1998-2003). París: UNESCO, 2003c.

UNESCO. Informe de síntesis sobre las tendencias y los avances en la educación superior desde la Conferencia Mundial sobre la Educación Superior (1998-2003). París: UNESCO, 2003d.

UNESCO. Conferencia mundial sobre la educación superior - 2009: la nueva dinámica de la educación superior y la investigación para el cambio social y el desarrollo. Paris: UNESCO, 2009. ED.2009/CONF.402/2

UNESCO.CEPES. Informe sobre las tendencias y los avances de la educación superior en Europa (1998-2003). Paris: UNESCO-CEPES, 2003. 
